\title{
Electron Irradiation Damage of MgO Nanocube
}

\author{
Huairuo Zhang, ${ }^{*}$ Marek Malac, ${ }^{, * *}$ and Ray F. Egerton, ${ }^{* * *}$ \\ *National Institute for Nanotechnology, Edmonton, Canada, T6G 2M9 \\ ** Department of Physics, University of Alberta, Edmonton, Canada, T6G 2G7
}

The irradiation effect of ceramics has been intensively studied for many years due to the important applications of ceramics in irradiation environments. As one of the most important ceramic materials, $\mathrm{MgO}$ received special attention in the fields of high energy ionic radiation and surface science [1,2]. In our work, the electron beam irradiation damage of $\mathrm{MgO}$ is studied in-situ with PEELS in a HF-3300 CFEG-TEM at an acceleration voltage of $300 \mathrm{KV}$. The $\mathrm{MgO}$ TEM sample was prepared by burning the $\mathrm{Mg}$ strip in the air and the smoke was collected on the holey carbon TEM grid.

Figure 1 shows TEM micrographs of $\mathrm{MgO}$ nanocubes suspended across a hole of carbon film, aggregated together by sharing the planes or edges. The dark speckles originate from the surface contamination during exposure in the air. The edge contrast reverses through focus as predicted by the projected charge density (PCD) approximation [3]. A single nanocube was selected with a [001] orientation to obtain uniform thickness along the electron beam direction and convergent-beam low-loss spectra collected to monitor irradiation effects. Mass loss was characterized in terms of relative thickness ( $\mathrm{t} / \lambda$ ), as shown in Figs. 2(a) and 2(b). The linear fits suggest mass loss by electron-beam sputtering and reveal a reduction about $28 \%$ when the $\mathrm{MgO}$ cube is on a carbon support than when $\mathrm{MgO}$ is in vacuum. However, the relative thickness increased and the fine structure in the $\mathrm{MgO}$ spectrum was gradually covered by the broad carbon plasmon peak, showing that carbon contamination can overwhelm mass loss due to irradiation.

Figure 3(a) shows a set of $\mathrm{MgO}$ valence-loss spectra collected on one nanocube, all normalized to the relative thickness $(t / \lambda)$. The energy resolution measured from the full width at half maximum of zero-loss peak is $0.55 \mathrm{eV}$. A slight red-shift of the plasmon peak (around $22 \mathrm{eV}$ ) was observed in the damaged $\mathrm{MgO}$ nanocube. In addition, as shown in Figure 3(b), some weak peaks between $3.8 \mathrm{eV}$ and $6 \mathrm{eV}$ appeared with increasing electron dose, regardless of whether the $\mathrm{MgO}$ nanocube was supported by a carbon film or suspended in vacuum. Figure 3(c) obtained from the pure amorphous carbon (a-C) film shows $\pi$-plasmon peak around $5 \mathrm{eV}$ appears apparently only when the thickness above $0.4 \mathrm{t} / \lambda$. The weak peaks in damaged $\mathrm{MgO}$ may have the contribution of carbon contamination; however, there are also contributions from oxygen vacancies (color centers) [4]: $\mathrm{F}^{+}$and $\mathrm{F}$ centers contribute to peak around $4.8-5.9 \mathrm{eV}$, while, $\mathrm{M}^{+}$and $\mathrm{M}$ centers appear around $3.9-4.7 \mathrm{eV}$. The peaks can not be attributed to Cerenkov radiation as they were observed only in damaged $\mathrm{MgO}$ and not observed in pristine $\mathrm{MgO}$ at both $300 \mathrm{kV}$ and $100 \mathrm{kV}$. 


\section{References:}

[1] S.J. Zinkle, C. Kinoshita, J. Nucl. Mater. 251 (1997) 200.

[2] J. Kramer, W. Ernst, C. Tegenkamp, H. Pfnür, Sur. Sci. 517 (2002) 87.

[3] J.C.H. Spence, High-Resolution Electron Microscopy, Third Edition, Oxford University Press Inc., New York, 2003.

[4] D. Domínguez-Ariza et al., Phys. Rev. B 68 (2003) 054101.
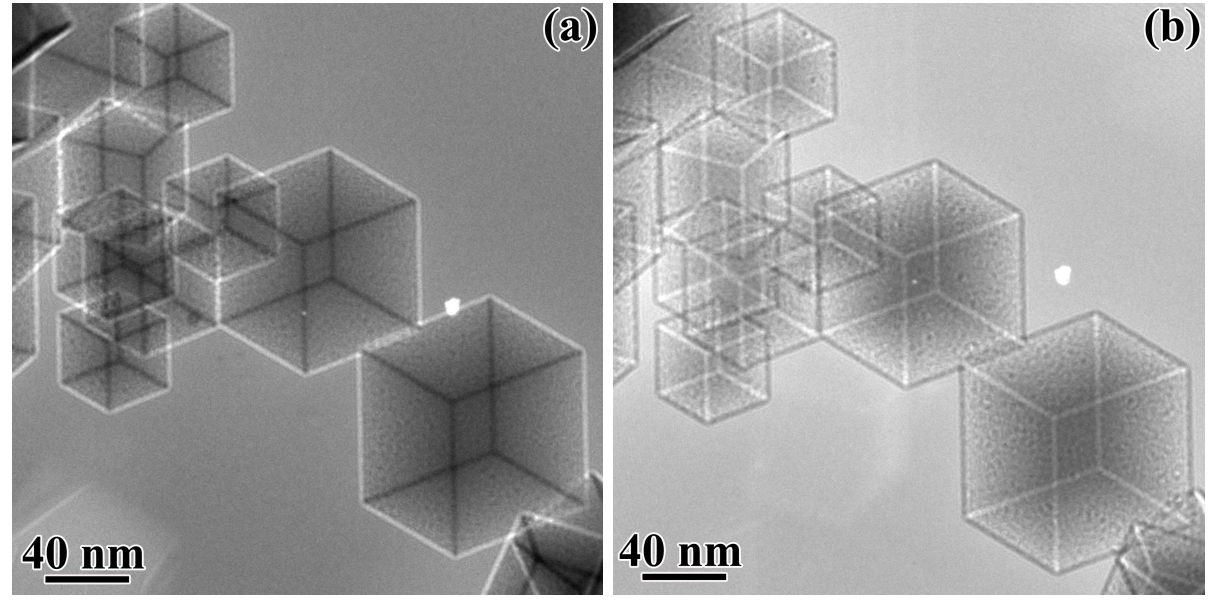

Fig. 1. (a) Under-focus and (b) over-focus TEM micrographs of $\mathrm{MgO}$ nanocubes suspending in the hole of the holey carbon grid, the white speckle in the image is a dust contamination in the CCD camera.
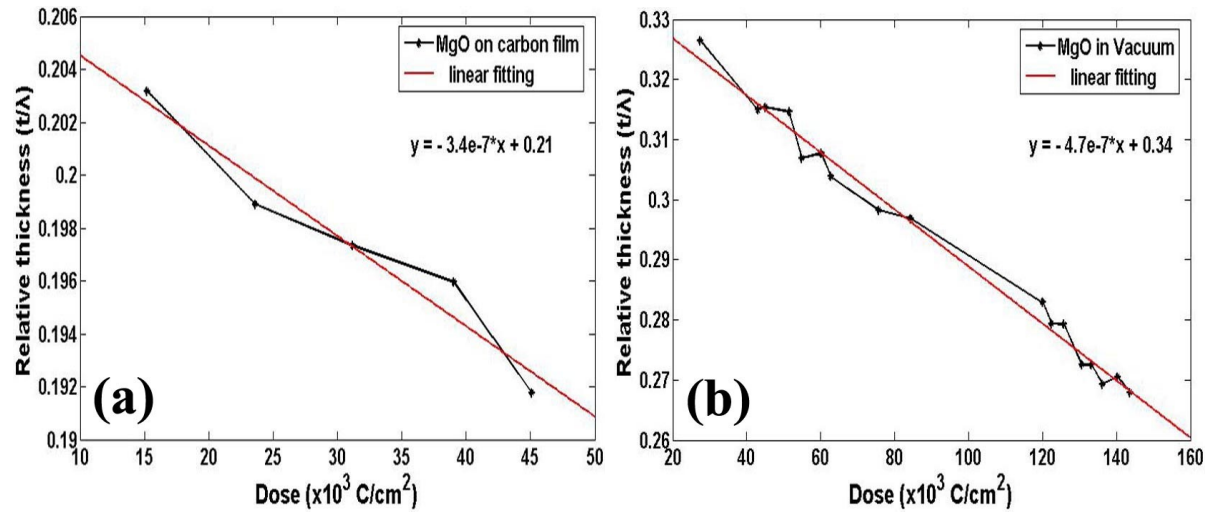

Fig. 2. The reduction of $\mathrm{MgO}$ nanocube relative thickness as a function of electron irradiation dose, measured for a $\mathrm{MgO}$ cube (a) on the carbon support and (b) in vacuum.

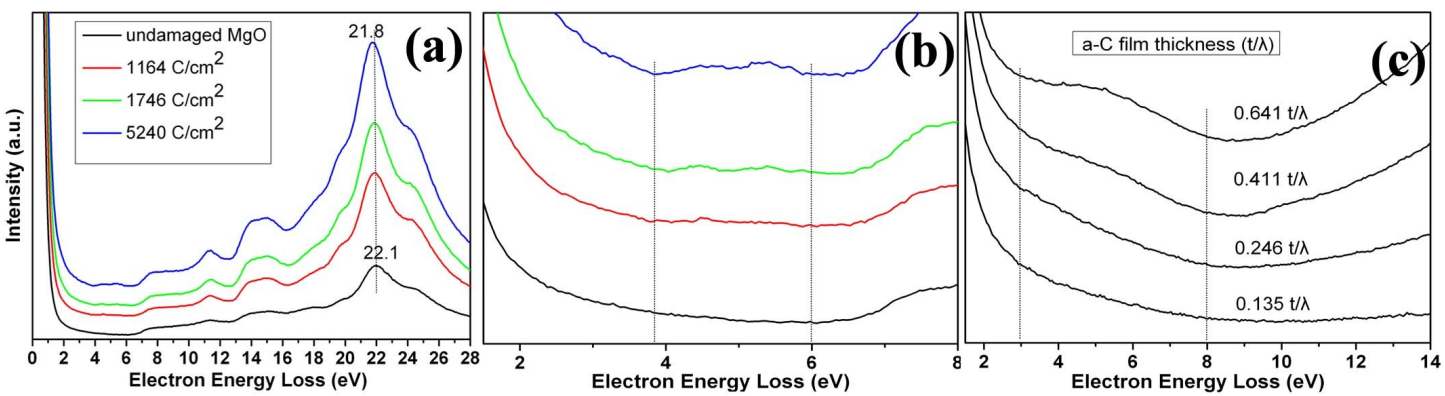

Fig. 3. (a) A set of low-loss spectra from an electron-irradiated $\mathrm{MgO}$ nanocube, (b) locally enlarged spectra, showing emerging fine structure, and (c) low-loss spectra of pure amorphous carbon film showing the $\pi$-plasmon peaks. All spectra are normalized to relative thickness $(\mathrm{t} / \mathrm{\lambda})$. 